\title{
Anthracycline-based triplets do not improve the efficacy of platinum-fluoropyrimidine doublets in first-line treatment of advanced gastric cancer: real-world data from the AGAMEMON National Cancer Registry
}

\author{
A. Carmona-Bayonas ${ }^{1} \cdot$ P. Jiménez-Fonseca ${ }^{2} \cdot$ A. Custodio $^{3} \cdot$ M. Sánchez Cánovas ${ }^{1}$. \\ R. Hernández ${ }^{4}$ - C. Pericay ${ }^{5}$ - I. Echavarria ${ }^{6}$ - A. Lacalle $^{7}$ - L. Visa ${ }^{8}$. \\ A. Rodríguez Palomo ${ }^{9} \cdot$ M. Mangas $^{10} \cdot$ J. M. Cano ${ }^{11} \cdot$ E. Buxo ${ }^{12} \cdot$ F. Álvarez-Manceñido ${ }^{9}$. \\ T. García ${ }^{1}$ - J. E. Lorenzo ${ }^{4}$ - M. Ferrer-Cardona ${ }^{5}$ - A. Viudez ${ }^{7}$ - A. Azkarate Az $^{13}$ \\ A. Ramchandani ${ }^{14}$ D. Arias ${ }^{15}$ - F. Longo ${ }^{16}$ - C. López ${ }^{17}$ - R. Sánchez Bayona ${ }^{18}$. \\ M. L. Limón ${ }^{19}$ - A. Díaz-Serrano ${ }^{20}$ A. Fernández Montes ${ }^{15}$ - P. Sala ${ }^{18}$.

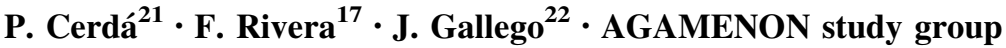

Received: 7 February 2017/Accepted: 3 April 2017/Published online: 9 April 2017

(C) The International Gastric Cancer Association and The Japanese Gastric Cancer Association 2017

\begin{abstract}
Background Although anthracycline-based triplets are one of the most widely used schedules to treat advanced gastric cancer (AGC), the benefit of including epirubicin in these therapeutic combinations remains unknown. This study
\end{abstract}

Electronic supplementary material The online version of this article (doi:10.1007/s10120-017-0718-5) contains supplementary material, which is available to authorized users.

A. Carmona-Bayonas

alberto.carmonabayonas@gmail.com

1 Hematology and Medical Oncology Department, Hospital Universitario Morales Meseguer, IMIB, Avenida Marqués de los Vélez, 30008 Murcia, Spain

2 Medical Oncology Department, Hospital Universitario Central de Asturias, Oviedo, Spain

3 Medical Oncology Department, Hospital Universitario La Paz, Madrid, Spain

4 Medical Oncology Department, Hospital Universitario de Canarias, Tenerife, Spain

5 Medical Oncology Department, Corporació Sanitària Parc Taulí, Sabadell, Spain

6 Medical Oncology Department, Hospital Universitario Gregorio Marañón, Madrid, Spain

7 Medical Oncology Department, Complejo Hospitalario de Navarra, Pamplona, Spain

8 Medical Oncology Department, Hospital Universitario Del Mar, Barcelona, Spain

9 Pharmacy Department, Hospital Universitario Central de Asturias, Oviedo, Spain aims to evaluate both the efficacy and tolerance of triplets with epirubicin vs. doublets with platinum-fluoropyrimidine in a national AGC registry.

Methods Patients with AGC treated with polychemotherapy without trastuzumab at 28 hospitals in Spain between 2008 and 2016 were included. The effect of anthracyclinebased triplets against doublets was evaluated by propensity

10 Medical Oncology Department, Hospital Galdakao-Usansolo, Galdakao-Usansolo, Spain

11 Medical Oncology Department, Hospital General de Ciudad Real, Ciudad Real, Spain

12 Medical Oncology Department, Hospital Universitario Clinic, Barcelona, Spain

13 Medical Oncology Department, Hospital Universitario Son Espases, Mallorca, Spain

14 Medical Oncology Department, Hospital Universitario Insular de Gran Canaria, Las Palmas de Gran Canaria, Spain

15 Medical Oncology Department, Complejo Hospitalario de Orense, Orense, Spain

16 Medical Oncology Department, Hospital Universitario Ramón y Cajal, Madrid, Spain

17 Medical Oncology Department, Hospital Universitario Marqués de Valdecilla, Santander, Spain

18 Medical Oncology Department, Clínica Universidad de Navarra, Pamplona, Spain

19 Medical Oncology Department, Hospital Universitario Virgen del Rocío, Seville, Spain 
score matching (PSM) and Cox proportional hazards (PH) regression.

Result A total of 1002 patients were included (doublets, $n=653$; anthracycline-based triplets, $n=349$ ). The multivariable Cox PH regression failed to detect significantly increased OS in favor of triplets with anthracyclines: HR 0.90 (95\% CI, 0.78-1.05), $p=0.20035$. After PSM, the sample contained 325 pairs with similar baseline characteristics. This method was also unable to reveal an increase in OS: 10.5 (95\% CI, 9.7-12.3) vs. 9.9 (95\% CI, 9.2-11.4) months, HR 0.91 (CI 95\%, 0.76-1.083), and (log-rank test, $p=0.226$ ). Response rates (42.1 vs. $33.1 \%$, $p=0.12$ ) and PFS (HR 0.95, CI 95\%, 0.80-1.13, log-rank test, $p=0.873$ ) were not significantly higher with epirubicin-based regimens. The triplets were associated with greater grade 3-4 hematological toxicity, and increased hospitalization due to toxicity by $68 \%$. The addition of epirubicin is viable, but $23.7 \%$ discontinued treatment because of adverse effects or patient decision.

Conclusion Anthracyclines added to platinum-fluoropyrimidine doublets did not improve the response rate or survival outcomes in patients with AGC but entailed greater toxicity.

Keywords Anthracyclines - Epirubicin .

Triplets:doublets · Gastric cancer · Stomach

\section{Introduction}

Standard therapy for advanced gastric cancer (AGC) that overexpresses or amplifies human epidermal growth factor receptor-2 (HER2) is the combination of trastuzumab with cisplatin and fluoropyrimidines [1]. However, this pathway is actived in only some $20 \%$ of the cases [2,3]. There are several treatment options for the remaining tumors (HER2 negative) that generally combine two or three cytostatics [4], with objective response rates of 35-45\% and median overall survival (OS) rarely exceeding 12 months [5].

Epirubicin-containing triplets currently comprise one of the most commonly used schedules, but whether associating this drug to the combination of platinum and fluoropyrimidine can increase efficacy or activity is unknown $[4,6]$. The role of 5-fluorouracil-cisplatin-epirubicin (ECF) in advanced disease was founded on a phase III trial that showed greater activity and survival, but less

20 Medical Oncology Department, Hospital Universitario Doce de Octubre, Madrid, Spain

21 Medical Oncology Department, Clínica Tecknon de Barcelona, Barcelona, Spain

22 Medical Oncology Department, Hospital General Universitario de Elche, Elche, Spain myelosuppression, compared to the 5-fluorouracil, doxorubicin, and methotrexate (FAMTX) schedule [7]. Nevertheless, the use of epirubicin as a single agent had previously demonstrated modest activity in AGC [8], and trials at the time were unable to confirm that adding anthracyclines was synergistic or contributed to enhancing the efficacy of 5-fluorouracil in monotherapy [9, 10].

Even at present, there are no phase III studies that compare FP vs. ECF, that is, the same schedules with and without anthracycline. The meta-analysis by Wagner et al. suggested increased OS for schedules with epirubicin: hazard ratio (HR) 0.77 , [95\% confidence interval (CI), 0.62-0.95], contributing to the more prevalent use of ECF [11]. However, it is worth mentioning that the trial with the greatest weight in this pooled measure included a comparison of two triplets, with and without epirubicin [12], whereas the other two trials had a very small sample size and hence did not allow for definitive conclusions to be reached $[13,14]$. As far as we know, only a randomized phase II trial $(n=91)$ has subsequently compared ECX against CX (cisplatin-capecitabine) regimens with progression-free survival as the primary endpoint, concluding that both were equivalent; hence, $\mathrm{CX}$ can be a reasonable standard chemotherapy [15].

Despite all this, the doubts around choosing platinum and fluoropyrimidine doublets or adding anthracyclines to them to obtain a triplet remain unresolved. The questions as to the additive effect of epirubicin in combination are still open, given the difficulty extrapolating the data to clinical practice entails, since real-world patients are often older, frailer, and have more chronic comorbidities than highly selected clinical trial populations. Registry-based cohort studies can also address real-world safety concerns by examining serious toxicities and risk-benefit ratios in larger series of patients.

Because there is not a single phase III trial to assess the still clinically relevant effect of adding anthracyclines, we have undertaken this analysis in an attempt to examine the efficacy and tolerance of triplets with anthracyclines versus fluoropyrimidine and platinum doublets alone.

\section{Patients and methods}

\section{Patient selection criteria}

The patients are from the national AGAMENON study of consecutive cases, in which 28 Spanish sites participated. The study design, characteristics, method, and data quality criteria have been extensively reported previously [3, 4]. Briefly, the eligibility criteria include adult patients ( $\geq 18$ years) with histologically confirmed, unresectable or metastatic gastric, gastroesophageal junction (GEJ), or 
distal esophageal adenocarcinoma who received first-line chemotherapy. Only those patients treated with two drugs (a platinum and a fluoropyrimidine), or with 3 drugs when the combination included a platinum, fluoropyrimidine, and epirubicin were included.

The most relevant exclusion criteria were the absence of at least 3 months of follow-up (except for patients who died before 3 months), fewer than 6 months since completing some kind of eventual adjuvant or neoadjuvant therapy, and the presence of other synchronous tumors. Patients who received first-line trastuzumab were excluded.

\section{Variables}

Fifteen factors that could influence the selection of triplet with anthracyclines vs. doublets with fluoropyrimidine and platinum schedules were chosen: sex, age, hypoalbuminemia (albumin $<3.5 \mathrm{~g} / \mathrm{dl}$ ), Eastern Cooperative Group Performance Status (ECOG PS) scale $\geq 2$, primary tumor site (esophagus, gastroesophageal junction, stomach), $\geq 2$ Charlson chronic comorbidities, chronic heart disease, stage at diagnosis (unresectable, locally advanced vs. metastatic), surgery for primary tumor, previous perioperative treatment, presence of signet ring cells, Lauren classification (diffuse vs. others), histological grade, Her2 status, site of metastases, and year of first-line therapy. Tumor response was assessed by the local investigators by means of an ex profeso reevaluation of the radiological images or computed tomography taken approximately every 3 months, as per the RECIST 1.1 criteria. Dose intensity (DI) was defined as the amount of drug administered per unit of time, expressed as $\mathrm{mg} / \mathrm{m}^{2}$ weekly. Cumulative dose was defined as the total dose and reported as total $\mathrm{mg} / \mathrm{m}^{2}$ administered. Relative dose intensity (RDI) was considered to be the DI administered with respect to the planned dose intensity for each schedule (Supplementary Table 1 and 2). Overall survival (OS) was defined as the time from treatment initiation to death from any cause; progression-free survival (PFS) was defined as the time from the beginning of chemotherapy until tumor progression or death from any cause, with censoring of patients who are lost to follow-up.

\section{Statistics}

Univariate screening with the above-named variables was performed. Those that were significantly associated with OS $(p<0.10)$ were entered into a Cox proportional hazards $(\mathrm{PH})$ regression model for OS together with the treatment schedule (with or without anthracycline, binary coded). Second, we used propensity score matching (PSM), a method that projects the probability of treatment selection on a scalar score as a basis on which to generate a relatively balanced distribution of baseline variables for both types of treatment [16]. Thus, 'nearest neighbor matching without replacement,' ratio $1: 1$, and 'caliper width' of 0.20 were chosen to match the observations with or without anthracyclines. To assess the pairing diagnostics, standardized differences of covariate values were used; $<10 \%$ indicated a negligible difference and special attention was paid to assure that additional, unforeseen imbalances were not generated in the process [16-18]. Subsequently, survival was estimated by the Kaplan-Meier method for the samples paired using this procedure. Stratified (by PS quintiles) log-rank tests were used to estimate the effect size of anthracyclines [17]. If subjects' true hazard ratio (relative risk) with anthracycline-based triplets versus doublets was 0.77, as in Wagner's meta-analysis [5], 460 events would have to be observed to be able to reject the null hypothesis that the survival functions of both groups are similar, with a probability (power) of 0.80 and type I error associated to this test of 0.05 [19]. All statistical assessments were two-sided, and $p$ values $<0.05$ were deemed significant. Statistical analyses were performed using RStudio, including the 'MatchIt' and 'survival' packages [20-22].

\section{Results}

\section{Patients}

Between January 2008 and December 2016, 2169 patients were registered, of whom 1002 were evaluable for this analysis. Figure 1 illustrates the selection process. At the time of analysis, 825 deaths $(82.7 \%)$ had been recorded, with a median OS of 10.4 months [95\% confidence interval (CI), 9.9-11.1]. The schedules used in first-line chemotherapy are presented in Supplementary Tables 1 and 2. To summarize, a doublet with platinum-fluoropyrimidine was used in $653(65.1 \%)$ and a triplet with anthracyclines in 349 (34.8\%) subjects; the most common triplet was EOX in 283 patients $(81 \%$ of the total of anthracycline-based triplets), whereas the most frequently used doublets were FOLFOX-6 in 177 (27.1\%), CAPOX in $163(24.9 \%)$, and XP in $150(22.9 \%)$ patients. Table 1 shows the distribution of subjects' baseline characteristics stratified per treatment. The imbalance of certain variables that were systematically associated with the use of triplets with anthracyclines versus platinum-fluoropyrimidine doublets is apparent, such as ECOG PS 0-1, absence of chronic heart disease, $<2$ chronic comorbidities, locally advanced, unresectable tumors, absence of liver metastases, and diffuse or poorly differentiated tumors. 
Fig. 1 Flowchart of patients in the registry

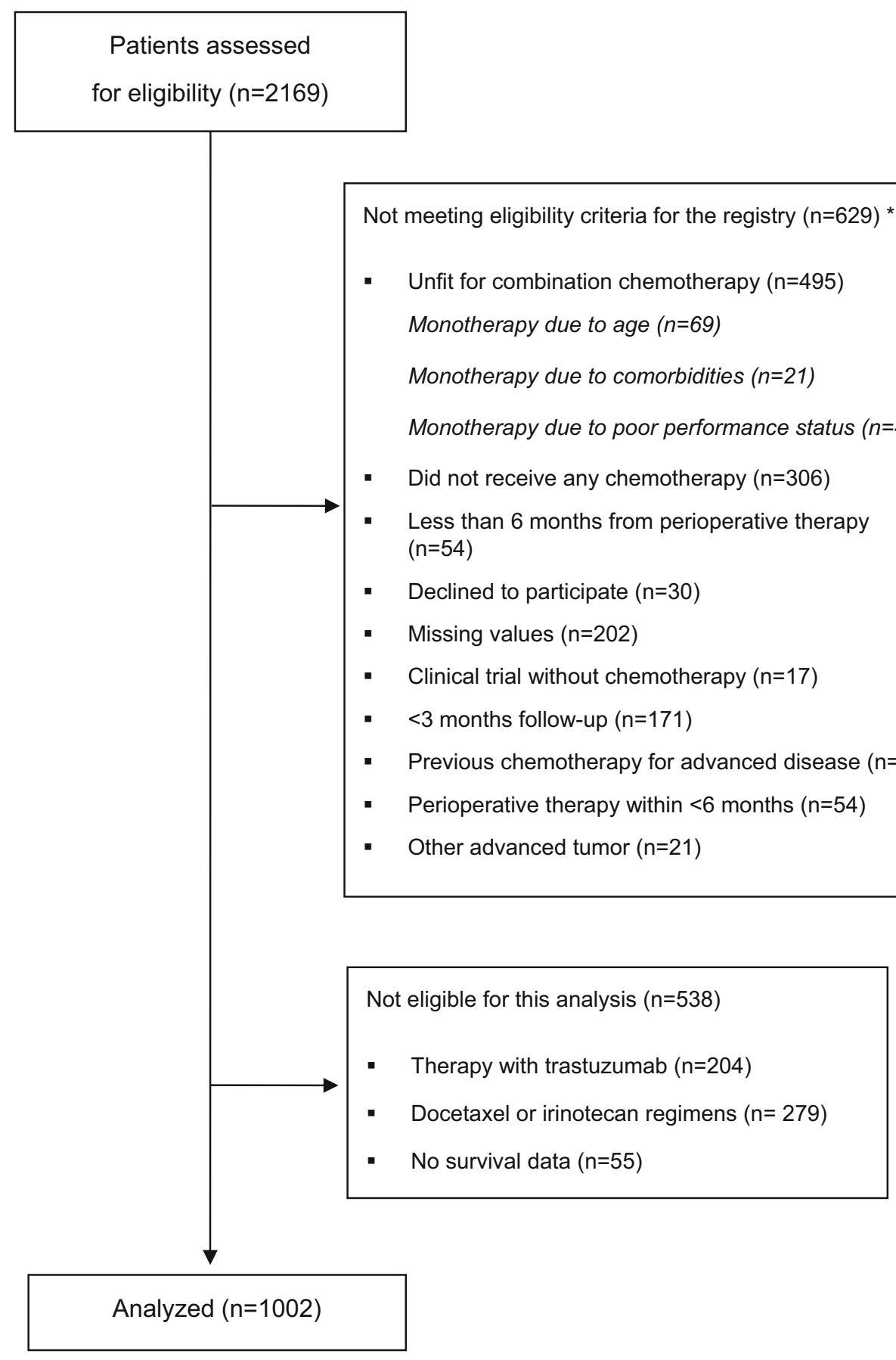

\section{Pre- and post-PSM patient characteristics}

In contrast, after implementing PSM, the study population consisted of 650 subjects ( 325 treated with schedules based on anthracyclines, evenly matched with another 325 treated with platinum and fluoropyrimidines doublets). Of them, 542 deaths $(83.3 \%)$ were recorded, with a median survival of 10.4 months (95\% CI, 9.6-11.4). Supplementary Figs. 1 and 2 illustrate the distribution of propensity scores before and after matching. Table 1 displays patients' baseline characteristics, with the standardized differences between variables, before and after PSM. The PSM procedure is seen to be effective in mitigating the standardized differences for all baseline characteristics and did not generate any additional imbalances. In particular, it is worth mentioning that a satisfactory balance was achieved of the differences between variables, such as ECOG PS, Her2 status, location of metastases, albumin, histological grade, or surgery for the primary tumor, which also significantly influenced prognosis in the Cox $\mathrm{PH}$ regression (see below). 
Table 1 Baseline characteristics of patients treated with triplets and doublets

\begin{tabular}{|c|c|c|c|c|c|c|}
\hline \multirow[t]{2}{*}{ Characteristics } & \multicolumn{3}{|l|}{ Before PSM } & \multicolumn{3}{|l|}{ After PSM } \\
\hline & $\begin{array}{l}\text { Platinum-based } \\
\text { doublet } n=653\end{array}$ & $\begin{array}{l}\text { Anthracycline-based } \\
\text { triplet } n=349\end{array}$ & $\mathrm{D}^{\mathrm{b}}$ & $\begin{array}{l}\text { Platinum-based } \\
\text { doublet } n=325\end{array}$ & $\begin{array}{l}\text { Anthracycline-based } \\
\text { triplet } n=325\end{array}$ & $\mathrm{D}^{\mathrm{b}}$ \\
\hline Male, female & $202(30.9 \%)$ & $111(31.8 \%)$ & 1.9 & $101(31.0 \%)$ & $101(31.0 \%)$ & 0 \\
\hline Age, mean \pm SD & $65.15 \pm 11.39$ & $59.6 \pm 11.87$ & 0.46 & $64.80 \pm 11.83$ & $59.53 \pm 11.95$ & -0.44 \\
\hline Albumin, $<3.5 \mathrm{~g} / \mathrm{dl}$ & $171(26.1 \%)$ & $93(26.6 \%)$ & 1.1 & $89(27.3 \%)$ & $86(26.4 \%)$ & -2.0 \\
\hline ECOG-PS $\geq 2$ & $124(18.9 \%)$ & $22(6.3 \%)$ & -38.6 & $26(8.0 \%)$ & $22(6.7 \%)$ & -4.9 \\
\hline$\geq 2$ Chronic comorbidities & $107(16.3 \%)$ & $27(7.7 \%)$ & -26.6 & $30(9.2 \%)$ & $27(8.3 \%)$ & -3.1 \\
\hline Chronic cardiopathy & $98(15.0 \%)$ & $21(6.0 \%)$ & -29.6 & $17(5.2 \%)$ & $21(6.4 \%)$ & 5.13 \\
\hline \multicolumn{7}{|l|}{ Primary tumor site } \\
\hline Esophagus & $44(6.7 \%)$ & $21(6.1 \%)$ & -2.4 & $16(4.9 \%)$ & $20(6.1 \%)$ & 5.2 \\
\hline $\begin{array}{l}\text { Gastroesophageal } \\
\text { junction }\end{array}$ & $66(10.1 \%)$ & $36(10.3 \%)$ & 0.6 & $36(11 \%)$ & $34(10.4 \%)$ & -1.9 \\
\hline Stomach & $543(83.2 \%)$ & $292(83.6 \%)$ & 1.0 & $273(84.0 \%)$ & $281(83.3 \%)$ & -1.8 \\
\hline $\begin{array}{l}\text { Stage at diagnosis, } \\
\text { metastatic }\end{array}$ & $634(97.0 \%)$ & $318(91.1 \%)$ & -25.1 & $309(95.0 \%)$ & $310(95.3 \%)$ & 1.3 \\
\hline $\begin{array}{l}\text { Surgery of the primary } \\
\text { tumor }\end{array}$ & $450(68.9 \%)$ & $212(60.7 \%)$ & -17.2 & $207(63.6 \%)$ & $204(62.7 \%)$ & -1.8 \\
\hline $\begin{array}{l}\text { Prior perioperative } \\
\text { treatment }\end{array}$ & $65(9.9 \%)$ & $37(10.6 \%)$ & 2.3 & $35(10.7 \%)$ & $36(11.0 \%)$ & 0.9 \\
\hline Signet ring cells & $209(32.0 \%)$ & $112(32.0 \%)$ & 0 & $115(35.3 \%)$ & $106(32.6 \%)$ & -6.7 \\
\hline $\begin{array}{l}\text { Lauren classification, } \\
\text { diffuse }\end{array}$ & $253(38.7 \%)$ & $137(39.2 \%)$ & 15.0 & $136(41.8 \%)$ & $130(40 \%)$ & -3.6 \\
\hline \multicolumn{7}{|l|}{ Histological grade } \\
\hline G1 & $73(11.2 \%)$ & $14(4.0 \%)$ & -27.4 & $12(3.6 \%)$ & $14(4.3 \%)$ & 3.5 \\
\hline $\mathrm{G} 2$ & $206(31.5 \%)$ & $93(26.6 \%)$ & -10.8 & $92(28.3 \%)$ & $93(28.6 \%)$ & 0.6 \\
\hline G3 & $251(38.4 \%)$ & $167(47.8 \%)$ & 19.0 & $146(44.9 \%)$ & $149(45.8 \%)$ & 1.8 \\
\hline Not available & $123(18.8 \%)$ & $75(21.4 \%)$ & 6.4 & $75(23.0 \%)$ & $69(21.2 \%)$ & -4.3 \\
\hline $\begin{array}{l}\text { Her2 positive }(3+, 2+ \\
\text { and } \mathrm{FISH}+)\end{array}$ & $20(3.0 \%)$ & $10(2.8 \%)$ & -1.1 & $8(2.4 \%)$ & $9(2.7 \%)$ & 1.9 \\
\hline \multicolumn{7}{|l|}{ Site of metastases } \\
\hline Liver & $254(38.8 \%)$ & $102(29.9 \%)$ & -18.8 & $113(34.7 \%)$ & $99(30.4 \%)$ & -9.1 \\
\hline Peritoneum & $292(44.7 \%)$ & $168(48.1 \%)$ & 6.8 & $153(47.0 \%)$ & $158(48.6 \%)$ & 3.2 \\
\hline Bone & $65(9.9 \%)$ & $35(10.0 \%)$ & 0.3 & $35(10.7 \%)$ & $34(10.4 \%)$ & -0.9 \\
\hline Lung & $55(8.4 \%)$ & $25(7.1 \%)$ & -4.8 & $19(5.8 \%)$ & $24(7.3 \%)$ & 6.0 \\
\hline Year of treatment, $\geq 2009$ & $620(94.9 \%)$ & $313(89.6 \%)$ & -19.9 & $302(92.9 \%)$ & $296(91.0 \%)$ & -6.9 \\
\hline
\end{tabular}

Percentages were calculated with respect to the columns

$P S M$ propensity score matching, $C I$ confidence interval, $D b$ standardized difference, ECOG-PS Eastern Cooperative Oncology Group Performance Status scale, $S D$ standard deviation, FISH fluorescence in situ hybridization

\section{Effect of epirubicin-based triplets versus doublets in the entire population}

In the Cox PH regression model, after adjusting for other prognostic covariates, subjects who received triplets with epirubicin were not seen to exhibit significantly higher OS, HR 0.90 (95\% CI, 0.78-1.05), $p=0.20035$, in comparison with those treated with platinum-fluoropyrimidine (see Table 2). Likewise, no difference was observed in PFS, with HR 0.97 (95\% CI 0.83-1.12), $p=0.708507$ (Supplementary Table 3$)$. In the binary logistic regression model, the use of anthracycline triplets was not associated with a significant increase in response rate: odds ratio 1.39 (95\% CI, 0.92-2.10), $p=0.11625$, after adjusting for other confounding variables (data not shown).

\section{Effect of adding epirubicin using the PSM-matched sample}

After obtaining an approximately balanced distribution of baseline variables, no increase in survival was detected: 10.5 (95\% CI, 9.7-12.3) vs. 9.9 (95\% CI, 9.2-11.4) months 
Table 2 Cox PH regression for overall survival in eligible patients $(n=1002)$
Fig. 2 Kaplan-Meier overall survival curves in subjects with platin-fluoropyrimidine doublets or anthracycline-based triplets, after PSM $(n=650)$. Median overall survival, 10.5 (95\% CI, 9.7-12.3) vs. 9.9 (95\% CI, 9.2-11.4) months (stratified logrank test, $p=0.226$ ), in patients who received triplets with epirubicin versus doublets, respectively, HR 0.91 (CI 95\%, 0.76-1.083). $\mathrm{Pl}$ platinum, $\mathrm{Fl}$ fluoropyrimidine, $E$ epirubicin, $P S M$ propensity score matching, $O S$ overall survival

\begin{tabular}{lrlll}
\hline Covariate & \multicolumn{1}{l}{$B$} & Hazard ratio (HR) & $95 \%$ CI of HR & $p$ value \\
\hline ECOG-PS $\geq 2$ & 0.47451 & 1.60723 & $1.3206-1.9561$ & $2.2 \mathrm{e}-06$ \\
$\geq 2$ Chronic comorbidities & 0.10621 & 1.11205 & $0.9070-1.3634$ & 0.30701 \\
No primary tumor surgery & 0.40946 & $1.50600^{\circ}$ & $1.2944-1.7522$ & $1.2 \mathrm{e}-07$ \\
Locally advanced tumors & -0.00133 & 0.99867 & $0.7106-1.4035$ & 0.99390 \\
Albumin, <3.5 g/dl & 0.26745 & 1.30663 & $1.1159-1.5300$ & 0.00089 \\
Lauren classification, diffuse & 0.00447 & 1.00448 & $0.8672-1.1634$ & 0.95248 \\
Her2 status, 3+ vs. others & -0.50658 & 0.60256 & $0.3757-0.9663$ & 0.03555 \\
Histological grade, grade 1 vs. others & -0.30889 & 0.73426 & $0.5666-0.9516$ & 0.01953 \\
Site of metastases & & & & \\
$\quad$ Peritoneum & 0.14050 & 1.15085 & $0.9959-1.3299$ & 0.05687 \\
$\quad$ Bone & 0.64371 & 1.90354 & $1.5234-2.3785$ & $1.5 \mathrm{e}-08$ \\
Anthracycline-based triplet & -0.09719 & 0.90738 & $0.7820-1.0529$ & 0.20035 \\
\hline
\end{tabular}

Schoenfeld's global test, applied to verify the proportional hazards assumption $\left(\chi^{2}=18.8, p=0.06348\right)$ $P H$ proportional hazards, $O S$ overall survival, $C I$ confidence interval, ECOG-PS Eastern Cooperative Oncology Group Performance Status scale

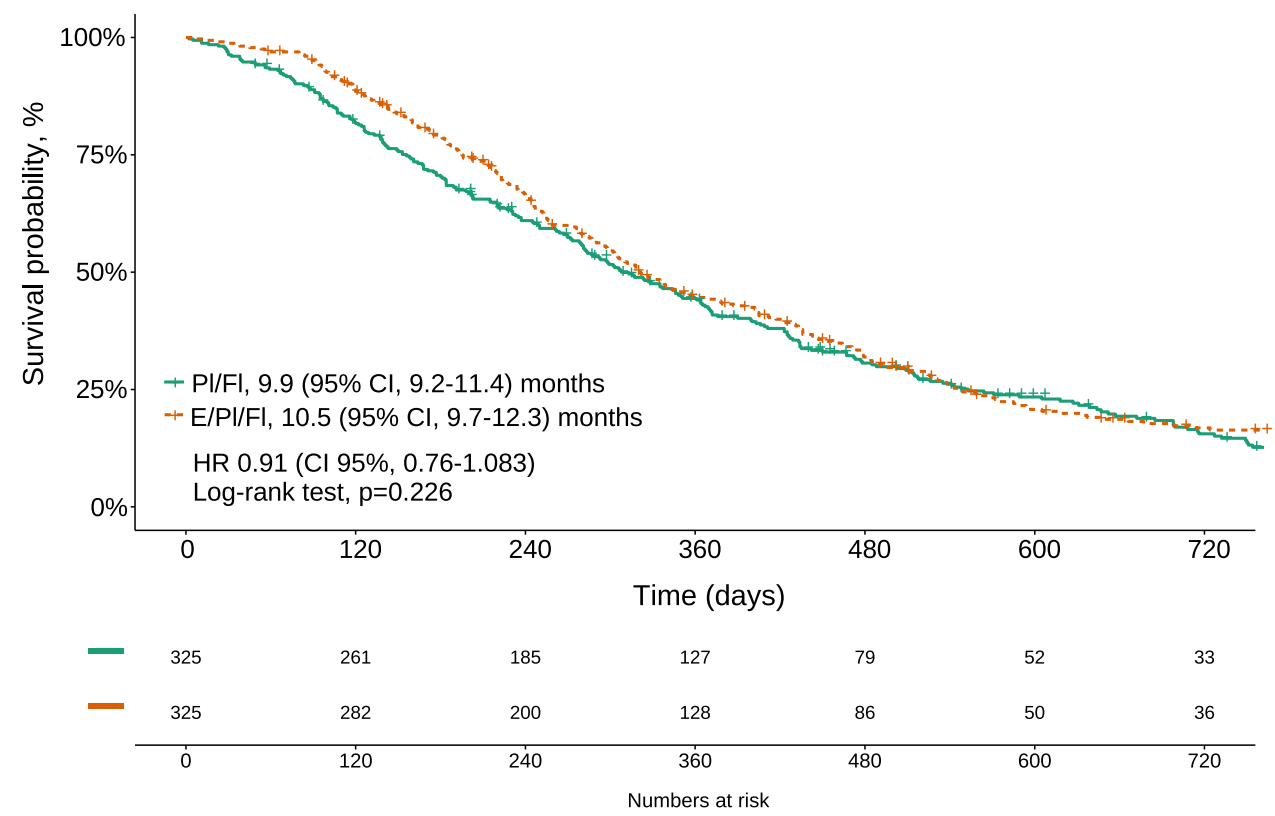

in patients who received triplets with epirubicin versus doublets, respectively, HR 0.91 (CI 95\%, 0.76-1.083), stratified log-rank test, $p=0.226$. The post-PSM KaplanMeier OS curves are shown in Fig. 2. Likewise, following PSM, no difference in PFS is noticed in favor of triplets containing anthracycline: 6.6 (95\% CI, 5.9-7.1) vs. 6.1 (95\% CI, 5.6-6.8) months, HR 0.95 (CI 95\%, 0.80-1.13), stratified log-rank test, $p=0.873$. The use of second lines of treatment is also similar for doublets and triplets with anthracyclines, 51.0 and $56.0 \%, p=0.208$, respectively. Resection of metastases was performed in 6.1 versus $7.0 \%$ for doublets in contrast to triplets, $p=0.752$. With respect to 3-month tumor response as per RECIST 1.1, a nonsignificant increase was found in the rate of objective responses with anthracycline-containing triplets as opposed to doublets: 42.1 vs. $33.1 \%, p=0.125$. Likewise, disease control rates (complete response, partial response, and stable disease) exhibited no statistically significant intergroup differences: 64.1 vs. $62.1 \%$, respectively, $p=0.724$.

Instead, the use of triplets with anthracyclines was associated with greater overall and grade 3-4 toxicity compared to platinum-fluoropyrimidine doublets, particularly more grade 3-4 anemia, neutropenia, febrile neutropenia (10.4 vs. 6.5\%), and toxicity-related hospitalization [31 vs. $18.4 \%$, odds ratio (OR) of 1.86 , (95\% CI, 1.27-2.73), $p=0.0007]$ (Table 3). These admissions took place after a median of 2 months of treatment in both groups. G-CSF prophylaxis was used in 
Table 3 Adverse events recorded after propensity score matching $(n=650)$

\begin{tabular}{|c|c|c|c|c|}
\hline \multirow[t]{2}{*}{ Toxicity } & \multicolumn{2}{|c|}{ Platinum-based doublet } & \multicolumn{2}{|c|}{ Anthracycline-based triplet } \\
\hline & Total $(\%)$ & Grade $3-4(\%)$ & Total $(\%)$ & Grade $3-4(\%)$ \\
\hline Anemia & 57.1 & 4.6 & 65.7 & 7.7 \\
\hline Neutropenia & 43.7 & 18.9 & 57.0 & 32.0 \\
\hline Febrile neutropenia & 5.5 & & 8.6 & \\
\hline Thrombocytopenia & 23.2 & 1.8 & 23.4 & 3.0 \\
\hline Emesis & 40.0 & 2.7 & 38.2 & 4.9 \\
\hline Diarrhea & 36.0 & 4.0 & 43.2 & 5.5 \\
\hline Stomatitis & 28.5 & 2.4 & 33.0 & 2.7 \\
\hline Fatigue & 69.8 & 6.8 & 66.9 & 5.5 \\
\hline Hand-foot syndrome & 26.0 & 0.3 & 27.1 & 3.0 \\
\hline Neuropathy & 52.4 & 3.4 & 66.0 & 4.0 \\
\hline Alopecia & 9.3 & & 61.7 & \\
\hline Increased aspartate aminotransferase & 10.5 & 0.3 & 16.3 & 0.9 \\
\hline Hyperbilirubinemia & 7.4 & 2.1 & 8.0 & 1.5 \\
\hline Renal toxicity & 5.9 & 0.3 & 6.7 & 0.6 \\
\hline Cardiotoxicity & 0.9 & 0.6 & 1.7 & 0 \\
\hline Venous thromboembolic disease & 11.4 & 4.0 & 11.1 & 6.7 \\
\hline Toxicity-related hospital admission & 18.4 & & 31.0 & \\
\hline Death due to toxicity & 0.6 & & 0.3 & \\
\hline
\end{tabular}

$23.6 \%$ of anthracycline-containing triplets vs. $12.9 \%$ of doublets $(p=0.0005)$.

\section{Doses used in triplets with anthracyclines or doublets in the PSM-matched sample}

Regarding the capacity to maintain the planned epirubicin dosing schedules in triplets, as well as the effect of adding this drug to the dose of platinum administered, there were essentially no differences in treatment duration, number of cycles or dose density, with the exception of a slight decrease in RDI of cisplatin of up to $77 \%$ for the XP schedule. The data do not support the hypothesis that the lack of incremental benefit from epirubicin-based triplets is due to the rapid reconversion in doublets or lowering of cytotoxic dosages, although in approximately one in four cases, the reason for discontinuing anthracycline was the emergence of toxicity deemed unacceptable or the patients' withdrawal. On the other hand, the most common causes leading to suspending epirubicin were the detection of tumor progression or completion of therapy because the maximum number of planned cycles had been administered (Table 4).

\section{Discussion}

In this article, we have analyzed the incremental effect of adding epirubicin to platinum-fluoropyrimidine doublets using the data from an observational study of AGC. After generating a subset of patients with an approximately homogeneous distribution of baseline characteristics, the anthracycline-containing triplets were not more efficacious in terms of objective response compared to doublets, and no significant differences in PFS or OS between groups were detected. In contrast, the use of anthracycline-containing triplets was associated with greater toxicity, which presumably impacts quality of life and healthcare resource use.

These results are certainly different from the conclusions of the meta-analysis by Wagner et al., who had previously reported a benefit in terms of OS associated with the use of anthracyclines-containing schedules, with a HR of 0.77 (95\% CI, 0.62 to 0.95) [11]. However, the results of this meta-analysis were based on three small, unequally designed trials, with comparators that are hardly commensurate to modern clinical practice. Furthermore, they were unable to directly respond to the question regarding the incremental effect of epirubicin and took place in a context of a striking increase in toxicity [12-14]. These outcomes from randomized controlled trials (RCTs) failed to resolve doubts as to the applicability of 'intensified' therapies in the real-world conditions of clinical practice of our study in which patients are often elderly, with impaired performance status, and a high percentage of whom suffer chronic comorbidities [23]. The paucity of data from appropriately designed RCTs with a suitable sample size is the main reason why this issue continues to be relevant even today, projecting itself in our 
Table 4 Doses of oxaliplatin, cisplatin, and epirubicin in frequent regimens (after PSM)

\begin{tabular}{|c|c|c|c|c|c|c|c|}
\hline \multirow[t]{2}{*}{ Doses for } & \multicolumn{3}{|c|}{ Oxaliplatin } & \multicolumn{3}{|c|}{ Cisplatin } & \multirow{2}{*}{$\begin{array}{l}\text { Epirubicin } \\
\text { EOX/ECF/ECX/EOF }\end{array}$} \\
\hline & EOX & FOLFOX6 & CAPOX & $\mathrm{XP}$ & FP3w & $\mathrm{ECX}$ & \\
\hline Number of cycles (median, range) & $6(1-13)$ & $8(1-16)$ & $5(1-15)$ & $5(1-9)$ & $6(1-6)$ & $6(1-12)$ & $6(1-12)$ \\
\hline Median of treatment duration (weeks) & 19 & 19 & 17 & 17 & 18 & 19 & 18 \\
\hline Mean cumulative dose $\left(\mathrm{mg} / \mathrm{m}^{2}\right)$ & 678 & 638 & 657 & 365 & 338 & 331 & 245 \\
\hline Mean dose/cycle $\left(\mathrm{mg} / \mathrm{m}^{2} /\right.$ cycle $)$ & 123 & 80 & 121 & 71 & 71 & 60 & 48 \\
\hline Mean dose intensity $\left(\mathrm{mg} / \mathrm{m}^{2} /\right.$ week) & 38 & 35 & 37 & 22 & 20 & 18 & 15 \\
\hline Mean, dose density & $87 \%$ & $84 \%$ & $86 \%$ & $77 \%$ & $80 \%$ & $90 \%$ & $88 \%$ \\
\hline \multicolumn{8}{|l|}{ Reason for withdrawal } \\
\hline Toxicity & $15.3 \%$ & $25.0 \%$ & $23.7 \%$ & $11.8 \%$ & $13.0 \%$ & $17.4 \%$ & $20.6 \%$ \\
\hline Progression & $39.2 \%$ & $28.0 \%$ & $46.1 \%$ & $51.5 \%$ & $30.4 \%$ & $34.8 \%$ & $33.6 \%$ \\
\hline Planned treatment completed & $34.3 \%$ & $23.0 \%$ & $17.1 \%$ & $27.9 \%$ & $43.5 \%$ & $17.4 \%$ & $34.5 \%$ \\
\hline Patient refusal & $1.1 \%$ & $5.0 \%$ & $2.6 \%$ & $1.5 \%$ & 0 & $17.4 \%$ & $3.1 \%$ \\
\hline Other & $7.5 \%$ & $16.0 \%$ & $6.6 \%$ & $4.4 \%$ & $4.3 \%$ & $13 \%$ & $7.7 \%$ \\
\hline Change to the ToGA regimen & 0 & 0 & $1.3 \%$ & 0 & 0 & 0 & 0 \\
\hline Not available & $2.6 \%$ & $3.0 \%$ & $2.6 \%$ & $2.9 \%$ & $8.7 \%$ & 0 & $0.6 \%$ \\
\hline
\end{tabular}

PSM propensity score matching; EOX epirubicin, oxaliplatin, capecitabine; FOLFOX6 fluorouracil, oxaliplatin; XP capecitabine, cisplatin; $C A P O X$ capecitabine, oxaliplatin; $D C X$ docetaxel, cisplatin, capecitabine; $D C F$ docetaxel, cisplatin, fluorouracil; $D O F$ docetaxel, oxaliplatin, fluorouracil; $D O X$ docetaxel, oxaliplatin, capecitabine

activity daily, debates, and research, which justifies conducting the analysis we present here [15].

In contrast, data from our study confirm those of a small randomized phase II study that compared the efficacy and safety of ECX vs. CX, basically with the same PFS and response rate that we report here [15]. However, we have found substantial differences in tolerance in our series with a higher rate of neutropenia, emesis and toxicity-related hospital admissions with anthracycline-based triplets.

In addition, our results are compatible with the indirect comparisons of a network meta-analysis recently reported by other authors who did not detect incremental benefits in OS and PFS for anthracycline-containing triplets compared to doublets based on fluoropyrimidines [6]. It is interesting to emphasize that the REAL-2 phase III clinical trial that led to the generalization of EOX in AGC confirmed the non-inferiority hypothesis for oxaliplatin and capecitabine versus cisplatin and fluorouracil; however, the study was not designed to appraise the value of adding epirubicin, since the four treatment arms included anthracycline [24]. The role of adding anthracyclines to platinum and fluoropyrimidine-based schedules is even more controversial if we consider that docetaxel-containing triplets have demonstrated their superiority to doublets not containing docetaxel in a multicenter, phase III study [25] and have achieved an increase in responses and a trend toward greater survival rates in a meta-analysis that included 12 RCTs [26]. Likewise, triplets with docetaxel have displayed a trend toward superiority over triplets with anthracyclines (NCT02445209) [27]. The conclusions of our study also differ to a certain extent from an earlier analysis presented by our own group, in which greater OS was observed in favor of the use of three-drug vs. two-drug schedules in general: HR, 0.77; 95\% CI, 0.65-0.92; stratified log-rank test, $p=0.004$. However, at that time, approximately one-third of the schedules were based on the addition of docetaxel as the third drug; consequently, the analysis could not be considered optimized to evaluate specifically the use of anthracyclines [28].

The uncertainty as to the real effect of anthracyclines might have negatively affected the development of new targeted drugs for AGC insofar as some research groups have assumed that epirubicin-based triplets (e.g., EOX or ECX) were both the scaffolding on which to add new molecules in phase III trials, as well as the standard comparator $[29,30]$. Thus, prior to scaling the risk-benefit of these kinds of triplets, the RILOMET- 1 and REAL- 3 trials already investigated the use of experimental quadruple therapies with the addition of monoclonal antibodies against MET and epidermal growth factor receptor (EGFR). This may have undermined the development of these targeted therapies, in part because the schedules were too toxic for patients $[29,30]$. In this regard, our analysis reveals that triplets with anthracyclines are viable in clinical practice, with high RDIs ( $>85 \%$ with respect to the planned dose) for the three components of the schedule. Nevertheless, close to one-fourth of the patients in our study discontinued treatment because of toxicity or because 
of their own decision, and, more importantly, the likelihood of patients' being hospitalized early because of grade 3-4 grade toxicity increased from 18.4 to $31.0 \%$.

This study has several study limitations inherent to evaluating effects in clinical practice studies. First, PSM is presumably capable of balancing an important part of the asymmetries in the baseline covariates, which results from the systematic selection of treatments in nonrandomized series. Thus, projecting the effect of numerous covariates on a scalar 'propensity score' entails the risk of generating unforeseen imbalances in the resulting sample. Despite the matching techniques used, it cannot be ruled out that some predictors such as performance status that simultaneously affect OS and triplet selection continue to behave as residual confounding factors. Second, the concept of 'doublet' or 'triplet' with or without epirubicin has covered several schedules. Insofar as it is doubtful that all schedules are truly equivalent in terms of efficacy or safety, the analysis must be contemplated as a perspective to be confirmed, ideally in RCTs. Third, most of the analyses we present are based on retrospective data with the limitations in accuracy inherent in studies of this kind, despite the fact that the main endpoint (OS), as well as the type of treatment and other measures, can be considered reliably collected, robust data. The reader must also be mindful that the effect size used for sample size determination derives from the results of a meta-analysis and therefore may not be optimal in a cohort with real-world data.

With all the afore-mentioned exceptions and in the absence of RCTs that improve the information presented herein, these results are of interest and can potentially be clinically applicable, in addition to generating hypotheses. Our data confirm the perception that, at least for most patients, the platinum-fluoropyrimidine doublet should be considered the initial standard treatment for AGC. The oncologists who participated in this study appear to have opted for the use of anthracycline-containing triplets vs. doublets in the case of patients with apparently more aggressive tumors (G3 or diffuse), perhaps with the justification of potentially greater efficacy in terms of response. However, the study does not evince any indication that benefitted in any noticeable way in clinical practice.

It is also doubtful that the use of triplets with anthracyclines should therefore be reserved for those special situations in which maximizing the possibility of objective tumor response could conceivably translate as a relevant clinical benefit (e.g., to alleviate a symptomatic patient or for surgery in a patient for whom resection is initially doubtful). While these circumstances are obviously conceivable, it is possible that the best choice in cases such as these might be a triplet containing docetaxel and not necessarily epirubicin [25-27].
In conclusion, the results of this study reveal that adding epirubicin to a combination of two drugs containing a platinum and fluoropyrimidine does not enhance the response rate, overall survival, progression-free survival, or clinical benefit. Furthermore, the epirubicin-containing triplet schedules used in our study incremented toxicity, the number of hospitalizations, burden of care for the teams, and, possibly, healthcare expenditure. Though this study presents the scientific limitations previously expounded, the data endorse the use of platinum- and fluoropyrimidinecontaining doublets in everyday clinical practice, except for special cases. Properly designed phase III trials with enough patients to draw conclusions with a greater level of evidence are needed. Nonetheless, we believe that those projects are quite unviable in western countries.

Acknowledgements We thank Priscilla Chase Duran for editing the manuscript, Natalia Cateriano, Miguel Vaquero, and IRICOM S.A. for supporting the registry website, and Ángel J. Lacave for the critical review of the manuscript and the interesting ideas provided.

\section{Compliance with ethical standards}

Conflict of interest The authors declare that they have no conflict of interest.

Funding None to declare; this is an academic study. The study was supported by the authors themselves.

Research involving human participants All procedures followed were in accordance with the ethical standards of the responsible committee on human experimentation (institutional and national) and with the Helsinki Declaration of 1964 and later versions. Informed consent or a substitute for it was obtained from all patients before they were included in the study.

\section{References}

1. Bang YJ, Van Cutsem E, Feyereislova A, Chung HC, Shen L, Sawaki A, et al. Trastuzumab in combination with chemotherapy versus chemotherapy alone for treatment of HER2-positive advanced gastric or gastro-oesophageal junction cancer (ToGA): a phase 3, open-label, randomised controlled trial. Lancet. 2010;376:687-97.

2. Tokunaga A, Onda M, Okuda T, Teramoto T, Fujita I, Mizutani $\mathrm{T}$, et al. Clinical significance of epidermal growth factor (EGF), EGF receptor, and c-erbb-2 in human gastric cancer. Cancer. 1995; 75:1418-25.

3. Jiménez-Fonseca P, Carmona-Bayonas A, Lorenzo MLS, Plazas JG, Custodio A, Hernández R, et al. Prognostic significance of performing universal HER2 testing in cases of advanced gastric cancer. Gastric Cancer. 2016. doi:10.1007/s10120-016-0639-8.

4. Carmona-Bayonas A, Jiménez-Fonseca P, Lorenzo MLS, Ramchandani A, Martínez EA, Custodio A, et al. On the effect of triplet or doublet chemotherapy in advanced gastric cancer: results from a National Cancer Registry. J Natl Compr Cancer Netw. 2016;14:1379-88. http://www.jncen.org/content/14/11/ 1379.abstract. Accessed 7 Apr 2017. 
5. Wagner AD, Unverzagt S, Grothe W, Kleber G, Grothey A, Haerting J, et al. Chemotherapy for advanced gastric cancer. The Cochrane database of systematic reviews (internet). 2016;CD004064. http://www.ncbi.nlm.nih.gov/pubmed/ 20238327. Accessed 7 Apr 2017.

6. Ter Veer E, Haj Mohammad N, van Valkenhoef G, Ngai LL, Mali RMA, Anderegg MC, et al. The efficacy and safety of firstline chemotherapy in advanced esophagogastric cancer: a network meta-analysis. J Natl Cancer Inst (internet). 2016;108. http://www.ncbi.nlm.nih.gov/pubmed/27576566. Accessed 7 Apr 2017.

7. Webb A, Cunningham D, Scarffe JH, Harper P, Norman A, Joffe $\mathrm{JK}$, et al. Randomized trial comparing epirubicin, cisplatin, and fluorouracil versus fluorouracil, doxorubicin, and methotrexate in advanced esophagogastric cancer. J Clin Oncol. 1997;15:261-7.

8. Coombes RC, Chilvers CE, Amadori D, Medi F, Fountzilas G, Rauschecker $\mathrm{H}$, et al. Randomised trial of epirubicin versus fluorouracil in advanced gastric cancer. An International Collaborative Cancer Group (ICCG) study. Ann Oncol (internet). 2016;5:33-6. http://www.ncbi.nlm.nih.gov/pubmed/8172789. Accessed 7 Apr 2017.

9. Kim NK, Park YS, Heo DS, Suh C, Kim SY, Park KC, et al. A phase III randomized study of 5-fluorouracil and cisplatin versus 5-fluorouracil, doxorubicin, and mitomycin $\mathrm{C}$ versus 5-fluorouracil alone in the treatment of advanced gastric cancer. Cancer (internet). 1993;71:3813-8. http://www.ncbi.nlm.nih.gov/ pubmed/8508349. Accessed 7 Apr 2017.

10. Cullinan SA, Moertel CG, Fleming TR, Rubin JR, Krook JE, Everson LK, et al. A comparison of three chemotherapeutic regimens in the treatment of advanced pancreatic and gastric carcinoma. Fluorouracil vs fluorouracil and doxorubicin vs fluorouracil, doxorubicin, and mitomycin. JAMA (internet). 1985;253:2061-7. $\quad$ http://www.ncbi.nlm.nih.gov/pubmed/ 2579257. Accessed 7 Apr 2017.

11. Wagner A, Grothe W, Haerting J, Kleber G, Grothey A, Fleig WE. Chemotherapy in advanced gastric cancer: a systematic review and meta-analysis based on aggregate data. J Clin Oncol. American Society of. Clin Oncol. 2006;24:2903-9.

12. Ross P, Nicolson M, Cunningham D, Valle J, Seymour M, Harper $\mathrm{P}$, et al. Prospective randomized trial comparing mitomycin, cisplatin, and protracted venous-infusion fluorouracil (PVI 5-FU) with epirubicin, cisplatin, and PVI 5-FU in advanced esophagogastric cancer. J Clin Oncol. 2002;20:1996-2004.

13. A randomized, comparative study of combination chemotherapies in advanced gastric cancer: 5-fluorouracil and cisplatin (FP) versus 5-fluorouracil, cisplatin, and $4^{\prime}$-epirubicin (FPEPIR). Kyoto Research Group for Chemotherapy of Gastric Cancer (KRGCGC). Anticancer Res (internet). 1992;12:1983-8. http:// www.ncbi.nlm.nih.gov/pubmed/1295444. Accessed 7 Apr 2017.

14. Kim TW, Choi SJ, Ahn JH, Bang HS, Chang HM, Kang YK, et al. A prospective randomized phase III trial of 5-fluorouracil and cisplatin (FP) versus epirubicin, cisplatin, and 5-fu (ECF) in the treatment of patients with previously untreated advanced gastric cancer (AGC). Eur J Cancer. 2001;37:S314.

15. Yun J, Lee J, Park SH, Park JO, Park YS, Lim HY, et al. A randomised phase II study of combination chemotherapy with epirubicin, cisplatin and capecitabine (ECX) or cisplatin and capecitabine (CX) in advanced gastric cancer. Eur $\mathrm{J}$ Cancer. 2010;46:885-91.

16. Austin PC. A critical appraisal of propensity-score matching in the medical literature between 1996 and 2003. Stat Med. 2008;27:2037-49.

17. Austin PC. A tutorial and case study in propensity score analysis: an application to estimating the effect of in-hospital smoking cessation counseling on mortality. Multivar Behav Res. 2011;46:119-51.

18. Austin PC. Balance diagnostics for comparing the distribution of baseline covariates between treatment groups in propensity-score matched samples. Stat Med. 2009;28:3083-107.

19. Schoenfeld DA. Sample-size formula for the proportional-hazards regression model. Biometrics. 1983;39(2):499-503.

20. Team R. RStudio: integrated development for R (2015). RStudio, Inc., Boston, MA. URL http://www.rstudio.com. Accessed 7 Apr 2017.

21. Ho DE, Imai K, King G, Stuart EA. MatchIt: Nonparametric Preprocessing for Parametric Causal Inference. J Stat Softw. 2011;42(8). https://www.jstatsoft.org/article/view/v042i08. Accessed 7 Apr 2017.

22. Therneau TM, Lumley T. Package 'survival'. 2016;2-37. http:// cran.um.ac.ir/web/packages/survival/survival.pdf. Accessed 7 Apr 2017.

23. Koppert LB, Janssen-Heijnen MLG, Louwman MWJ, Lemmens VEPP, Wijnhoven BPL, Tilanus HW, et al. Comparison of comorbidity prevalence in oesophageal and gastric carcinoma patients: a population-based study. Eur J Gastroenterol Hepatol. 2004;16:681-8.

24. Cunningham D, Starling N, Rao S, Iveson T, Nicolson M, Coxon F, et al. Capecitabine and oxaliplatin for advanced esophagogastric cancer. The New England journal of medicine. 2008;358(1):36-46.

25. Van Cutsem E, Moiseyenko VM, Tjulandin S, Majlis A, Constenla M, Boni C, et al. Phase III study of docetaxel and cisplatin plus fluorouracil compared with cisplatin and fluorouracil as firstline therapy for advanced gastric cancer: a report of the V325 Study Group. J Clin Oncol. 2006;24(31):4991-7.

26. Chen X-L, Chen X-Z, Yang C, Liao Y-B, Li H, Wang L, et al. Docetaxel, cisplatin and fluorouracil (DCF) regimen compared with non-taxane-containing palliative chemotherapy for gastric carcinoma: a systematic review and meta-analysis. PLoS One (internet). 2013;8:e60320. http://www.ncbi.nlm.nih.gov/pubmed/ 23593191.

27. Ochenduszko S, Puskulluoglu M, Konopka K, Fijorek K, Urbanczyk K, Budzynski A, et al. Comparison of efficacy and safety of first-line palliative chemotherapy with EOX and mDCF regimens in patients with locally advanced inoperable or metastatic HER2-negative gastric or gastroesophageal junction adenocarcinoma: a randomized phase 3 trial. Med Oncol. 2015;32:1-8.

28. Carmona-Bayonas A, Jiménez-Fonseca P, Lorenzo MLS, Ramchandani A, Martínez EA, Custodio A, et al. On the effect of triplet or doublet chemotherapy in advanced gastric cancer: results from a National Cancer Registry. J Natl Compre Cancer Netw internet). 2016;14:1379-88. http://www.ncbi.nlm.nih.gov/ pubmed/27799509.

29. Waddell T, Chau I, Cunningham D, Gonzalez D, Okines AFC, Wotherspoon A, et al. Epirubicin, oxaliplatin, and capecitabine with or without panitumumab for patients with previously untreated advanced oesophagogastric cancer (REAL3): a randomised, open-label phase 3 trial. Lancet Oncol. 2013;14:481-9.

30. Cunningham D, Tebbutt NC, Davidenko I, Murad AM, Al-Batran S-E, Ilson DH, et al. Phase III, randomized, double-blind, multicenter, placebo $(\mathrm{P})$-controlled trial of rilotumumab $(\mathrm{R})$ plus epirubicin, cisplatin and capecitabine (ECX) as first-line therapy in patients (pts) with advanced MET-positive (pos) gastric or gastroesophageal juncti. In: ASCO annual meeting proceedings. 2015. p. 4000 